\title{
Sylkan: Towards a Vulkan Compute Target Platform for SYCL
}

\author{
Peter Thoman \\ petert@dps.uibk.ac.at \\ University of Innsbruck \\ Innsbruck, Austria
}

\author{
Daniel Gogl \\ daniel.gogl@student.uibk.ac.at \\ University of Innsbruck \\ Innsbruck, Austria
}

\author{
Thomas Fahringer \\ tf@dps.uibk.ac.at \\ University of Innsbruck \\ Innsbruck, Austria
}

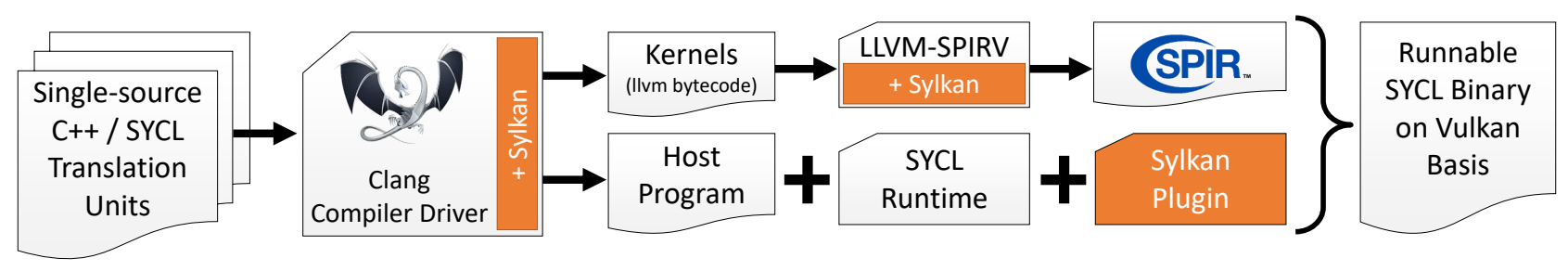

Figure 1: Overview of the Sylkan compilation process and the involved runtime components.

\begin{abstract}
SYCL is a modern high-level $\mathrm{C}++$ programming interface which excels at expressing data parallelism for heterogeneous hardware platforms in a programmer-friendly way, and is standardized by the Khronos Group. The latest version of the standard, SYCL 2020, removes the previous dependence of the specification and its implementations on an underlying OpenCL target, opening the door for compliant alternative implementations.

In this paper, we discuss the opportunities and challenges of mapping SYCL to Vulkan, a low-level explicit programming model for GPUs. This includes an analysis of the potential semantic mismatch between each respective standard, as well as approaches to work around some of these issues. Additionally, we present a prototype research implementation of Sylkan, a SYCL compiler and runtime targeting Vulkan.

In order to evaluate our prototype qualitatively and quantitatively, we chose a variety of functional tests as well as three performance benchmarks. For the functional tests, we discuss and categorize the failures of the current prototype, noting which semantic mismatch or missing implementation causes them. For the performance benchmarks, we compare execution times against a OpenCL-based SYCL implementation and a native Vulkan version of each benchmark, on two hardware platforms.
\end{abstract}

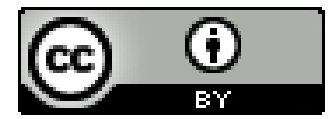

This work is licensed under a Creative Commons Attribution International 4.0 License. IWOCL'21, April 27-29, 2021, Munich, Germany

(c) 2021 Copyright held by the owner/author(s). Publication rights licensed to ACM. ACM ISBN 978-1-4503-9033-0/20/04 ..\$15.00

https://doi.org/10.1145/3456669.3456683

\section{CCS CONCEPTS}

- Computing methodologies $\rightarrow$ Massively parallel algorithms; Graphics processors; Parallel programming languages; • General and reference $\rightarrow$ Performance.

\section{KEYWORDS}

GPU computing, parallel languages, compilers, runtime systems, SYCL, Vulkan

\section{ACM Reference Format:}

Peter Thoman, Daniel Gogl, and Thomas Fahringer. 2021. Sylkan: Towards a Vulkan Compute Target Platform for SYCL. In International Workshop on OpenCL \& SYCL, Munich, Germany, April 27-29, 2021. ACM, New York, NY, USA, 12 pages. https://doi.org/10.1145/3456669.3456683

\section{INTRODUCTION}

With Moore's Law slowing down [5], end-users in many computational domains are turning to increasingly complex parallelization methods in order to see speedups in their workloads. One particularly promising avenue is heterogeneous computing, which leverages the high peak performance and energy efficiency of specialized, often highly parallel hardware - usually GPUs, or, more generally, accelerators - to implement suitable algorithms. This development has led to a proliferation of all kinds of parallel computing hardware, each with its own unique set of best practices and often a preferred set of development tools [6].

While these considerations may appear straightforward from a hardware-centric, parallelism expert perspective, in practical use the programmability of heterogeneous systems is a significant hindrance to their broader adoption in domain sciences [14] and industry. Targeting accelerators requires an accelerator-specific API and programming model, and the most widespread vendor-agnostic option, OpenCL [21], assumes familiarity with low-level hardware details and imposes significant implementation effort and maintenance overhead. While various vendor-specific options exist, and some of these offer a more high-level API $[2,16]$, the possibility of 
vendor lock-in makes them less attractive in many important use cases.

One option to mitigate these issues is implementing multiple backends targeting distinct architectures and their matching APIs. However, not only does this type of software stack impose maintenance challenges, it also reduces flexibility in performing more sweeping and algorithmic optimizations, and makes adapting a given program for future hardware architectures more difficult.

The Khronos SYCL standard [23] seeks to address this issue by providing a high-level programmability-focused C++ API for targeting heterogeneous devices. While the initial standard was explicitly dependent on an OpenCL runtime for target devices, SYCL 2020 [18] eliminates this dependence and thereby allows more flexible backend design.

As a result, there are already several SYCL 2020 implementations in the works, targeting traditional GPUs, HPC accelerators, FPGAs, and more. However, there is one category of devices which is still not adequately covered by any currently planned SYCL implementations that we are aware of: the GPUs in low-cost mobile and embedded SoCs. Frequently, no OpenCL drivers are provided for these devices, and no other general compute API for heterogeneous data parallelism is widely supported either.

The situation is quite different for another Khronos standard: Vulkan [19] is widely supported on almost all relevant consumer hardware due to its importance for end-user graphical applications and particularly games.

To allow an even larger set of hardware devices to be targeted using SYCL, we have created Sylkan, a compiler tooling and runtime system for executing SYCL programs on Vulkan devices. Sylkan is currently in a prototype state, but is already capable of running a variety of basic SYCL programs successfully. In this work we focus specifically on documenting the semantic gap between the existing SYCL design and software tools and the Vulkan ecosystem, with our central contributions comprising:

- An analysis of the semantic and engineering gap between SYCL and Vulkan, both in terms of their specifications and in terms of the current tool ecosystem, including a description of some of the more complex issues and their potential solutions.

- A prototype compiler and runtime implementation based on LLVM, which includes various compiler modifications and transformations as well as a SYCL runtime plugin targeting Vulkan.

- An evaluation of our prototype implementation from both qualitative and quantitative perspectives, compared to manual Vulkan and baseline SYCL-on-OpenCL implementations.

To the best of our knowledge, this work is the first to explore running SYCL on arbitrary Vulkan-capable devices.

The remainder of this paper is structured as follows. Section 2 provides important background information regarding SYCL and Vulkan, with a special focus on the relevant mapping from one API and runtime model to the other. In particular, section 2.4 discusses the mapping of several fundamental aspects of a SYCL program execution to Vulkan.

In section 3 we describe the current implementation of the Sylkan prototype, split into its compile-time parts in section 3.1 and the runtime components in section 3.2. Section 4 analyses and illustrates the results of our evaluation. We provide a qualitative result by checking the ability of our prototype to run various feature tests in section 4.3. In terms of performance evaluation, we have manually implemented three basic kernels in SYCL and Vulkan and measured their relative performance in section 4.2. Finally, an overview of related work in section 5 precedes the conclusion of this paper.

\section{BACKGROUND AND SEMANTIC MAPPING}

In this section we provide a short overview of both SYCL and Vulkan, including their respective standards as well as some information related to their tool ecosystems, especially as it relates to the goals and implementation of Sylkan. We then describe the methodology we chose to semantically map from SYCL to Vulkan at a high level. A more low-level implementation-focused description follows in section 3 .

\subsection{SYCL}

SYCL was originally released in 2015 [10] with the goal of serving as a high-level, cross-vendor API for implementing high-performance algorithms on heterogeneous parallel computing platforms. The initial 1.2 standard [23] relied on OpenCL as its implementation vehicle, with various interoperability aspects required for conformance. It later became clear that it is preferable to loosen this constraint [1], and the provisional SYCL 2020 specification allows for more freedom in backend implementations [18].

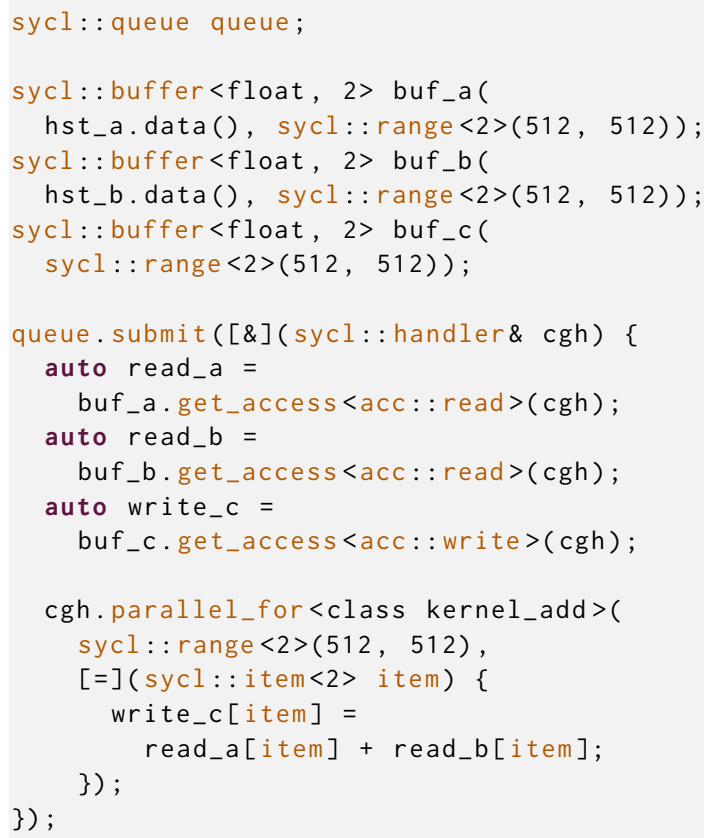

Listing 1: A simple SYCL program which adds two buffers.

The core concepts of SYCL that developers interact with to construct their algorithms are $\mathrm{N}$-dimensional buffers and kernels. The former store data, and the latter are enqueued in some work queue to asynchronously perform computations on this data. In listing 1 , 
three 2D buffers are created in lines 3-8, and a kernel is submitted to an execution queue in lines $10-24$.

A crucial characteristic of SYCL, which sets it apart from many other languages in the field, is the fact that developers are not required to explicitly encode a dependency graph between their kernels. Instead, a SYCL program records the data requirements of each kernel by means of accessors, and the runtime system automatically provides potentially asynchronous scheduling of computations and memory transfers based on this information. Lines 11 to 16 in listing 1 define three accessors, two for reading from the buffers $a$ and $b$, and one for writing to c. During program execution this information is recorded, and if e.g. another kernel were to be enqueued subsequently which reads from c, a dependency on kernel_add would be created automatically.

The second essential feature of SYCL, especially from the perspective of compiling for a new backend, concerns its code structure. Unlike OpenCL or most compute languages in traditional graphicsfocused APIs such as OpenGL [20] and DirectX [15], SYCL implements a single-source model. As shown in listing 1, general host code (e.g. buffer allocation) and specialized kernel code (e.g. lines 20-23) exist in the same source file, but might ultimately be executed by two entirely distinct hardware units, requiring a compilation toolchain capable of recognizing the device and host parts of the program, and generating code for each.

While the entirety of SYCL is offered as a $\mathrm{C}++$ library, forming an embedded DSL, kernel code is required to fulfill a set of constraints which limit the $\mathrm{C}++$ base language to a subset of features amenable to device compilation. In the current SYCL standard [23], these restrictions are detailed in section 6.3. They relate primarily to two categories:

Memory spaces and allocation - prohibiting the direct transfer of pointer-based data structures, default heap memory allocation, use of the $\mathrm{C}++$ thread storage duration, and access to non-constant globals.

Control flow - disallowing recursion, virtual dispatch, and calls via function pointers.

Additionally, some individual runtime features such as RTTI are also not available in kernel code. All of these constraints extend to any application or library code and data structures transitively used within kernels.

\subsection{Vulkan}

Vulkan [24] is a low level, cross-vendor and cross-platform API designed to allow expert programmers to leverage the full performance potential of massively parallel graphics hardware. To that end, it offers a very explicit interface, which leaves all details of memory management, synchronization and orchestration of host and device code execution to the developer. While this means that non-experts might require additional software layers to build upon instead of interacting with Vulkan directly, it also makes the API very suitable in principle as a basis for implementing higher-level constructs - such as SYCL.

Vulkan supports general computation by means of compute kernels, which need to be supplied in SPIR-V [22] form at runtime. For illustration purposes, listing 2 shows GLSL kernel code which implements functionality similar to the previous SYCL example, and can be compiled to SPIR-V. Note that a linear index has to be computed manually, unlike in SYCL where 2D indexing is natively supported by buffer accessors. It should also be noted that the SYCL code sample corresponds to a complete (excerpt) of a program, containing both host and device code. On the other hand, for Vulkan we only show the compute Kernel - all the required data structures still need to be allocated and bound in a corresponding host program.

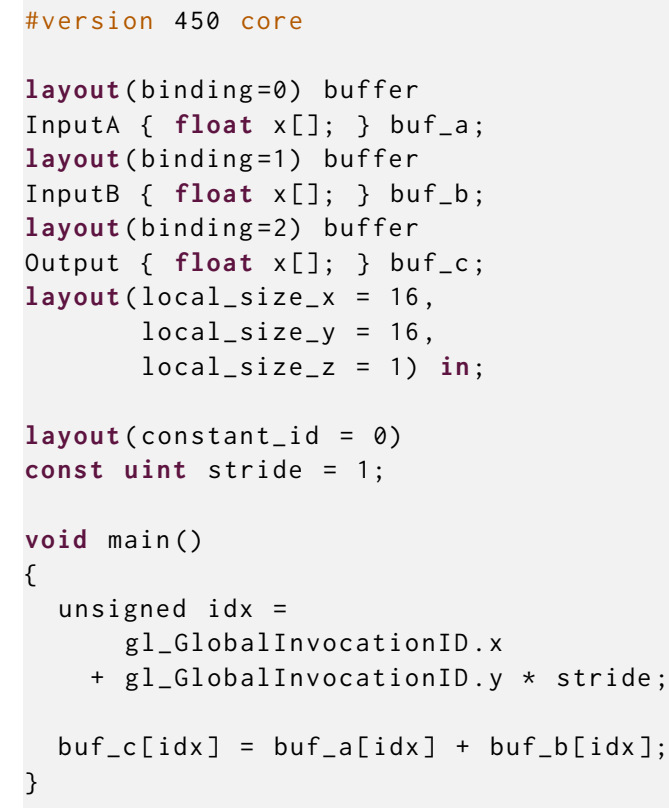

Listing 2: GLSL compute kernel code which implements behaviour similar to listing 1 .

More specifically, this host work entails allocating the required resources, and creating a pipeline which matches up the kernel with its parameters using their corresponding binding IDs. Data transfers between host and device memory also need to be staged and synchronized manually, while they happen implicitly in the SYCL sample. The full host code required for this purpose goes beyond the scope of this discussion, but this difference serves as an illustration of the benefits of being able to target Vulkan compute devices with a higher level of abstraction.

\subsection{Tool Ecosystems}

Attempting a medium- to large-scale software engineering effort in a vacuum is difficult at best, in particular under the constraints of academic research. As such, when developing Sylkan we sought to re-use as much existing infrastructure as possible. Luckily, as a very widespread industry standard, a wealth of tooling exists for Vulkan, and the recent renewed interest in SYCL 2020 has lead to a greatly improved tool situation, especially regarding open source projects. We will now provide a quick overview in order to explain and justify our choices for the underlying technology used in Sylkan.

Table 1 summarizes the most important and widely used SYCL implementations currently available, including their intended target 


\begin{tabular}{l||l|l|l} 
Implementation Name & Primary Dev. & Target Platforms & Source Availability \\
\hline \hline DPC++ & Intel & CPUs, OpenCL+SPIR-V, CUDA+PTX & Yes, LLVM-based \\
ComputeCpp & Codeplay & CPUs, OpenCL+SPIR, OpenCL+PTX & No \\
hipSYCL & Uni. Heidelberg & OpenMP, ROCm, CUDA & Yes, LLVM-based \\
triSYCL & Xilinx & OpenMP, OpenCL+SPIR & Yes
\end{tabular}

Table 1: Summary of the most mature currently available SYCL implementations.

platforms and source availability. DPC $++{ }^{1}$ is an extended branch of LLVM maintained by Intel, ComputeCPP ${ }^{2}$ is a commercially supported implementation by Codeplay, hipSYCL ${ }^{3}$ was developed as a research project [1] but now provides a mature platform particularly for GPUs, and triSYCL ${ }^{4}$ is a more research-focused and experimental open source implementation.

As previously explained, Vulkan consumes SPIR-V as its shader input language, and as such an open source implementation targeting SPIR-V was the most sensible option for our purpose. Therefore, we chose the $\mathrm{DPC}++$ compiler and runtime system as the basis for the implementation of Sylkan.

\subsection{Semantic Mapping}

Before we discuss individual aspects of the semantic mismatch between SYCL and Vulkan, we first want to clarify the used language. While both are Khronos standards, SYCL terminology was influenced significantly by OpenCL and fundamental objects and their semantics largely correlate between the two. However, the Vulkan API uses a different object structure and terminology in some cases - table 2 shows a possible semantic mapping of both domains.

The most interesting choice here is mapping Events to Timeline Semaphores. While not strictly necessary for a working SYCL implementation, timeline semaphores support omnidirectional synchronization on both host and devices using a single primitive, and thereby make the implementation significantly cleaner and easier to extend and troubleshoot in our experience. This choice may limit the target platforms for Sylkan in the very short term, as timeline semaphores were only promoted from an extension to a core feature in Vulkan 1.2. However, we expect them to become widely available in the near future, once implementors adopt the new specification.

Kernel Code. Both OpenCL 2.1 and Vulkan consume device code in the SPIR-V format, and as explained in section 2.3, the base technology we chose for implementing Sylkan already generates kernels in this intermediate language. For anyone not familiar with the details of SPIR-V, this could give the impression that there is only a small or even nonexistent semantic mismatch, and little work beyond adapting aspects such as parameter passing needs to be done to enable SYCL kernels running on a Vulkan execution backend from a compiler perspective. However, in practice we found that kernel code compilation actually poses the most significant

\footnotetext{
${ }^{1}$ https://github.com/intel/llvm/tree/sycl

${ }^{2}$ https://developer.codeplay.com/products/computecpp/ce/home

${ }^{3}$ https://github.com/illuhad/hipSYCL

${ }^{4}$ https://github.com/triSYCL/triSYCL
}

\begin{tabular}{c|c} 
SYCL / OpenCL & Vulkan \\
\hline Platform & Instance \\
Device & Physical Device \\
Context & Device \\
Queue & Queue \\
Buffer & Buffer \\
Program & Shader Module \\
Kernel & (Compute) Pipeline \\
Event & Timeline Semaphore
\end{tabular}

Table 2: Object mapping from SYCL to Vulkan.

challenge, and the reasons for this relate to the details of which dialect of SPIR-V is accepted in different APIs.

The SPIR-V format supports a wide range of capabilities. At the start of each SPIR-V file a list of all needed capabilities must be declared in order to allow certain features and instructions to be used within the code. And here we encounter a very significant difference: on the one hand OpenCL 2.1 can readily consume SPIR-V code with the kernel and addresses capabilities, while on the other hand Vulkan only supports the shader capability. The major effect of this capability disparity is that a valid SPIR-V kernel for OpenCL 2.1 cannot be consumed by a Vulkan program - and vice versa. In the interest of brevity, for the remainder of this paper we now define shaders and kernels as SPIR-V code valid for Vulkan and SPIR-V code valid for OpenCL 2.1, respectively, in any context where we compare or contrast the two.

We have derived the following main categories of differences between kernels and shaders from their disparate support of SPIR-V capabilities:

Addressing Model Kernels allow a rather feature-rich physical addressing model, but shaders are constrained to a more limited logical addressing model. The logical addressing model severely restricts the use of pointers, prohibiting, among other features, the casting of pointers and their use with a generic address space.

Address Spaces The storage class CrossWorkgroup is the default for OpenCL global memory. Although the SPIR-V specification does not explicitly prevent using CrossWorkgroup in shaders, it is not featured in the Vulkan specification's list of allowed storage classes. However, the storage class 
StorageBuffer has generally equivalent properties and can be used in shaders, which is our approach in Sylkan.s

Calling conventions In OpenCL 2.1, kernels can be viewed as functions and their arguments are variables or buffers which are passed directly to the kernel. In shaders, by definition, the "kernel" function must not take any arguments. Data is passed to the function by means of global variables of structure type, and is bound by the API on the host side using descriptor sets. These structures are allowed to have at most one runtime array element as their final member variable. In Sylkan, runtime arrays enable us to pass buffers with variable sizes into shaders.

Structured control flow The Vulkan API requires structured control flow in shaders, the properties of which are discussed in-depth in the SPIR-V specification. Beyond affecting the permitted control-flow graph (CFG) structure, it additionally requires the inclusion of explicit merge instructions at branching operations. There are two types of these: loop branch instructions and selection branch instructions. A selection branch is needed for if or switch constructs while a loop branch is needed for any loop construct.

Each of those constructs contains a header block and exactly one matching merge block. Rules note that a single block must not be a merge block to multiple header blocks, and that a header block is defined by holding a merge instruction (either OpMerge for loop constructs or OpSelection for selection constructs) before the leaving branch instruction. This further implies that a single block cannot be a header block for a loop and a header block for a selection at the same time.

While these categories encompass the major discrepancies affecting kernel code and its interaction with the host program, there are some other semantic differences of note which also need to be accounted for in Sylkan.

User Code Structure and Runtime. As previously discussed, an important feature of SYCL is its support for single-source applications targeting heterogeneous platforms. Vulkan, on the other hand, follows a traditional split host and device code model. Therefore, to implement SYCL on Vulkan a compiler driver is required to extract the relevant kernel code and separately compile it for the device target architecture. Fortunately, as detailed in the implementation section, the Clang frontend in the $\mathrm{DPC}++$ base technology we are using already accomplishes this task and only required minor extensions for Sylkan.

Finally, a very significant runtime difference between SYCL and Vulkan is how they handle dependencies. Vulkan is entirely explicit and leaves synchronization to the user, while SYCL - at least in its default use case with accessors - automatically tracks dependencies and performs synchronization as well as data transfers. Fortunately the SYCL runtime for DPC++ is sufficiently generic to enable different backends while leveraging the same dependency tracking code. This allowed us to primarily focus on mapping the individual mechanics of building block operations such as memory allocation, transfers, and synchronization to Vulkan, while leaving the orchestration of these operations up to the generic runtime system.

\section{SYLKAN IMPLEMENTATION}

Figure 1 provides a high-level overview of the Sylkan compilation process as well as the runtime components involved, and serves as an overarching guide for the discussion of our prototype implementation in this chapter. The entire system leverages several components from existing toolchains, which we modified and extended for our needs. The majority of these modifications were necessary in the Clang compiler and LLVM-SPIRV, which are marked in the flowchart. Additionally, the Sylkan backend plugin for the SYCL runtime is, of course, entirely new as part of this work. Our Sylkan research prototype is open source software and available on Github ${ }^{5}$.

\subsection{Compile Time}

The open-source DPC++ compiler, serving as a basis for Sylkan, contains a working toolchain for creating SPIR-V code valid in OpenCL 2.1. Section 3.1.1 describes its compilation process, including cooperating tools and shows how the are connected. The subsequent sections will focus on the main differences between kernels and shaders pointed out in section 2.4, and note which program parts had to be adapted to enable valid shader code generation.

3.1.1 DPC ++ Compiler Overview. The DPC ++ compiler separates host and device code and compiles them in separate steps. In SYCL, the device code is identifiable by functors passed to certain methods of the command group handler (e.g. parallel_for). The code invoked in the operator () of these functors is compiled to functions in the LLVM intermediate representation. All captured variables will become arguments to these functions. Special objects such as SYCL accessors are simplified, passed as several arguments and reconstructed on the device side by the _-_init method. This requires that all these object classes contain an _- init method in order to derive the necessary parameters passed to the kernel function from its arguments. E.g. an accessor will be passed as four parameters: (i) data pointer, (ii) access range, (iii) memory range, and (iv) offset. With this information it is possible to calculate the linear index for given multidimensional indices on the device side. Note that the sizes of the ranges and offset depend on the compile-time dimensionality of the accessor.

As a side product, the device code compilation also generates an integration header which includes kernel names and their signatures. This header is automatically included in the host code compilation process.

The result of the device code front end compilation is a binary in LLVM intermediate representation. The next step is converting this LLVM IR into device code. Since the Intel Clang compiler supports multiple SYCL backends, it is possible to have various device code compilers. In the case of OpenCL 2.1, it requires SPIR-V code which can be generated by the LLVM-SPIRV bidirectional translator. Luckily LLVM IR and SPIR-V have a rather similar structure and share many operations. Therefore, it is possible for the LLVM-SPIRV translator to convert one LLVM IR operation into one or multiple SPIR-V operations linearly.

\footnotetext{
${ }^{5}$ https://github.com/tadeaustria/llvm/tree/syclcon2021
} 


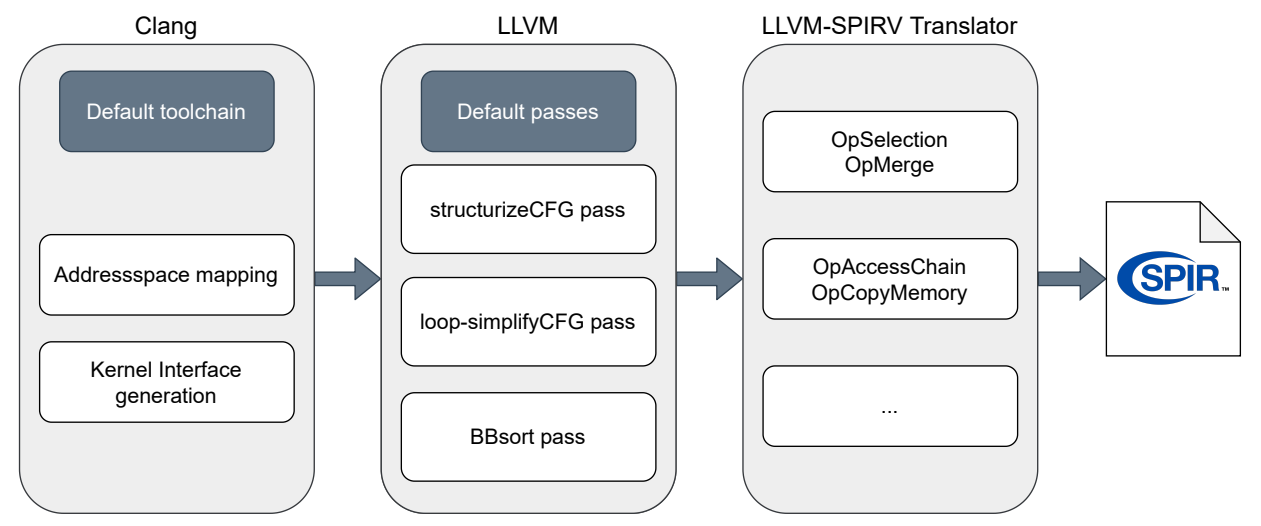

Figure 2: An overview of the device code compilation process, highlighting its adaptions for our Sylkan implementation.

Once the device code is generated, the host code, together with the corresponding integration header as well as the SYCL API headers can be compiled like any standard host program. Afterwards the device code (e.g. SPIR-V) is bundled together with the compiled host program and finally linked with other translation units and the SYCL runtime library resulting in a single fat binary executable.

3.1.2 Sylkan Compilation. In this section we will provide more details on the various adaptations and extensions to the $\mathrm{DPC}++$ compilation process that were necessary for our Sylkan prototype. As illustrated in fig. 2, three major components of the compilation toolchain required various modifications: in the Clang frontend, the address space mapping and kernel interface generation steps were adapted; during the core LLVM compilation phase, three additional passes were introduced; and in the LLVM-SPIRV translator, a large number of mapping operations were (re-)implemented specifically for generating SPIR-V code with shader semantics.

Note that this overview and the following descriptions are not exhaustive, but instead focus on the most important structural changes. For example, we also made some minor modifications to existing LLVM passes such as instCombine in order to generate valid shader binaries.

Addressing Model and Pointers. Device code optimizations and in particular function call inlining reduce the overall addressing problem, since indirect function calls are often eliminated. Therefore, optimization passes are generally necessary for correct Sylkan device code generation. However, there are some pointer calculations and casts introduced by LLVM IR which cause issues. The LLVM IR instruction getelementptr is used to navigate into structures and calculate indices in arrays. E.g. the $C$ expression $a+5[3]$, where $a$ is an integer array, will result in the access of the $(5+3=8)$ 9th element. In LLVM IR without optimization this results in the following simplified statements: $\% 0=$ getelementptr a 5 and $\% 1$ $=$ getelementptr $\% 03$ where $\% 1$ is then the pointer to the 9 th element.

For SPIR-V code which targets kernel capabilities a directly equivalent instruction is available - OpPtrAccesschain - which the LLVM-SPIRV translator generates in such situations. Unfortunately, this instruction is not allowed for shaders. The alternative OpAccessChain instruction is quite similar but the input pointer allows only objects of composite type. Because an array is a composite type, it is possible to access any of its elements using OpAccessChain. However, the pointer to e.g. the 6th element of an integer array is not a composite type anymore and cannot be incremented by three to access the 9th element by using OpAccessChain. This means that the previously shown example of step-wise pointer calculation is not possible in Vulkan SPIR-V. Therefore the LLVM-SPIRV translator was modified to backtrack along possible getelementptr chains, accumulate their indices and create equivalent results. E.g. if both element accesses are needed in the preceeding sample, the following SPIR-V code would be generated: $\% 0=$ OpAccessChain a 5 and $\% 1=$ OpAccessChain $a$ 8.

Another related topic is the handling of memory copy operations in LLVM IR. memcpy only allows byte pointers as input and the length has to be passed in. Again the equivalent instruction available to OpenCL 2.1 SPIR-V OpCopyMemorySized is not available to shaders. SPIR-V offers a slightly more abstract instruction OpCopyMemory which deduces the required byte size from the type of its argument pointers. However, for this purpose these pointers need to have the original type. As such, the LLVM-SPIRV translator was modified to eliminate many instances of casts to byte pointers and use the original pointers for copy operations.

Address Spaces. Address spaces are usually not dealt with explicitly in most normal programs targeting CPUs, but for efficiency of GPU and accelerator computation they play an important role. In SPIR-V address spaces are called storage classes and combined with types they participate in semantic type checking. For instance, an integer pointer to global memory is a different type than an integer pointer to local memory. SPIR-V also introduces the generic storage class for polymorphic usage, however, this is prohibited in shaders.

From the SYCL perspective, address spaces are a result of the buffers users access, and need to be passed down through front end Clang compilation and SPIR-V translation into the final SPIRV code. SYCL features the access: : target type which defines four different storage classes: global, local, constant and private. Clang implements these address spaces as type attributes e.g. -attribute__((addrspace(ID))), where ID can be either an identifier for built-ins or a constant integer. In LLVM IR the address 
space is just an integer value without any further semantic meaning. However, type consistency regarding address spaces is still required. Finally, the LLVM-SPIRV translator converts the integer values from LLVM IR into storage class information for SPIR-V (Mapping table 'SPIRSPIRVAddrSpaceMap' in SPIRVInternal.h).

From the Sylkan perspective, one problem of generated OpenCL SPIR-V code is that all global variables default to the storage class CrossWorkgroup instead of the Vulkan-compatible StorageBuffer class. To utilize Clang's type and address space inference, we found that the most efficient implementation strategy is to replace the address space in an early stage of compilation. Therefore, the mapping table used by Clang was replaced for Sylkan, such that the built-in opencl_global address space maps to the StorageBuffer class.

Additionally, when accesses are performed through an accessor, address space information may be lost. As a result, in LLVM IR all accesses to global accessors are cast to normal pointers. Since address space casts are not allowed in shaders, it was necessary to modify the accessor compilation behaviour in order to expose the address space on usage and eliminate address space casting.

Another related topic is the address space of built-in variables. Those are also mapped to the prohibited CrossWorkgroup storage class. In this case, it proved sufficient to add the __attribute _( (addrspace (7)) ) qualifier for built in variables in the SYCL runtime headers, which then maps to the Input storage class required by Vulkan SPIR-V for this purpose.

Calling Conventions. The intuitive calling convention where kernels can be seen as functions with parameters does not have a direct equivalent in the Vulkan API for compute shaders. Here, parameters need to be passed through structural global variables and data has to be bound with descriptor sets. For the device code this means that instead of creating functions with parameters, these parameters need to be wrapped into structure types and a member expression must be inserted at each usage. Code generation for this purpose was injected in Clang for Vulkan SPIR-V target device code compilation.

A challenge in this context is that the code generation for parameters can have a wide range of requirements and can depend on parameter types. The plugin below the SYCL runtime, however, has very limited knowledge about the semantics of each parameter. The plugin interface is heavily influenced by the first plugin and original SYCL backend for OpenCL. In OpenCL, there are only two types of parameter passing, either a variable or a pointer to allocated device memory. For SYCL accessors, the data pointer is passed as allocated device memory and its other components are passed as variables, with each constituting a separate call of the plugin. Within each call the plugin is unaware that these parameters originally formed an accessor, which makes it hard to deal with special cases at the plugin level.

Here, passing parameters via global variables introduces a drawback: When multiple shaders are associated with a translation unit, they share the same global variable pool. At runtime, parameter meta-information is required to build an appropriate and complete descriptor set. Therefore, it was necessary to extend the integration header at compile time with parameter offset information. This

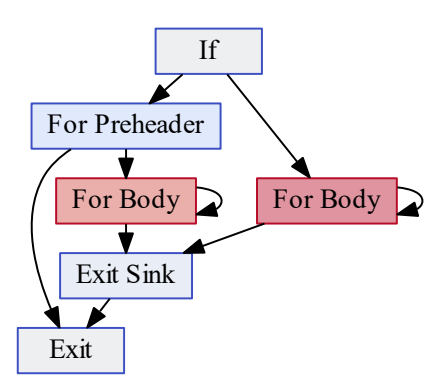

Figure 3: Kernel Control Flow Graph.

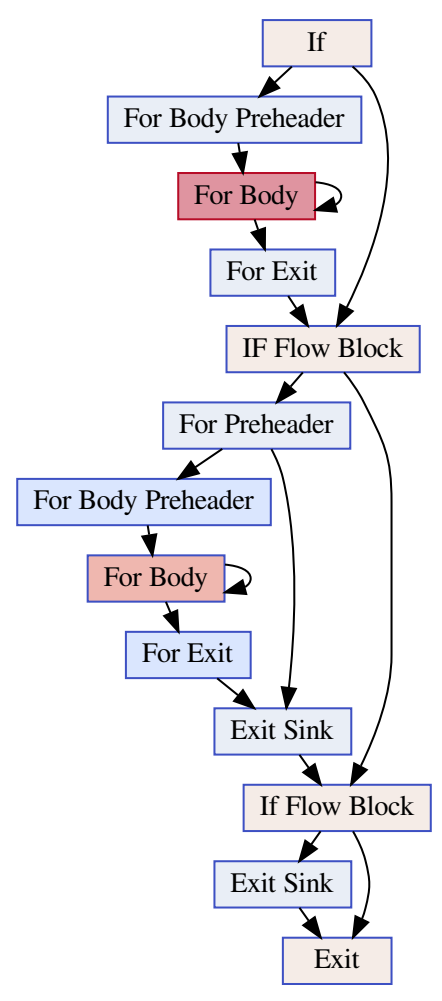

Figure 4: Shader Control Flow Graph.

allows the runtime to accurately index the parameters for each individual kernel.

The currently implemented concept of using individual buffers for all types of parameters keeps the implementation effort manageable. However, for achieving optimal performance it may be required to switch to specialization constants or push constants for non-buffer parameters in the future.

Structured Control Flow. As opposed to OpenCL SPIR-V kernel code, Vulkan-compliant SPIR-V needs to follow strict structured control flow rules. Every block containing multiple branching possibilities must be marked with the merge block where all possible branches eventually converge, forming a selection construct. We extended the LLVM-SPIRV translator to implement these structures 
by performing a dominator tree search for each conditional branch. The closest common successor is selected and used as a merge block by generating the OpSelection instruction.

Similarly, code containing loops and therefore a back edge in the control flow needs to follow the strict definition of a loop construct. A loop header must be marked with information of both the first block reached after exiting the loop and the unique block, which is allowed to have the only backedge to the loop header. The LLVM-SPIRV converter already features support for creating these constructs in SPIR-V code. Code comments for this feature claim that loop information generated by the LLVM loop-simplify pass is required. Unfortunately in our experience this information was not created despite activating the pass. For Sylkan we extended the SPIR-V generation step to use an LLVM pass which creates the missing information for the loop construct.

Even with these adaptations it is possible that the generated control flow does not follow rules for structured control flow. For instance, it is not allowed that a block is a merge block for two different headers. It may also occur that a loop header is the same block as a selection header, which is illegal. Luckily LLVM features passes for structuring control flow and loop simplification, which create explicit exit blocks for each construct.

We leveraged these existing passes, however, some of the newly created blocks might be inserted after their respective successor, which is in violation of another SPIR-V rule. A newly created LLVM pass, BBSort, topologically rearranges strongly connected components in a SPIR-V compatible way.

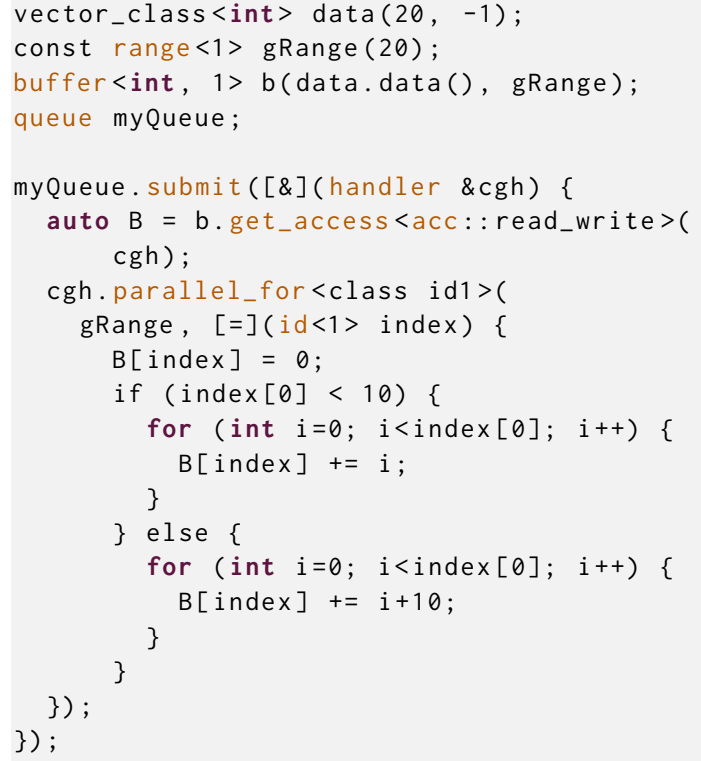

Listing 3: Kernel requiring CFG transformations for Vulkan.

Unfortunately the LLVM structurize control flow pass does not fully address the specific problem of loop and selection header overlap. We solved this issue in the LLVM-SPIRV translator by detecting this type of structure and inserting a simple block only containing the original conditional branch and selection construct.
The loop header is adapted to refer to this new block, and block related instructions in all successors are updated accordingly.

To better illustrate the impact of all of these control flow adaptations we have included a small example. Listing 3 shows SYCL code for a basic kernel exhibiting relatively interesting control flow, extracted from one of our test cases. Figure 3 illustrates the original control flow graph on the LLVM IR level without any Sylkan processing, while Figure 4 shows the final shader CFG after the processing steps outlined above. Additional flow blocks and sinks were constructed to fulfill all requirements of SPIR-V structured control flow.

\subsection{Runtime}

3.2.1 DPC++ Runtime. The DPC++ runtime is separated into two major components, the core SYCL runtime and backend plugins. The former implements all required SYCL logic (e.g. buffer handling and maintaining call dependencies), while plugins contain calls to underlying backend APIs, which are needed to communicate with target devices. Currently available plugins include OpenCL, Level Zero and CUDA.

Compiling device codes for multiple plugins and gathering all of these versions in a single fat binary file enables the flexibility to defer the backend decision to the runtime. To support this use case, the Intel SYCL runtime reads the SYCL_DEVICE_FILTER environment variable which allows pattern-based backend selection

3.2.2 Vulkan Plugin. The design of the DPC++ SYCL runtime plugin interface appears to be derived from the OpenCL API, with several functions being mapped directly. However, for our newly created Vulkan plugin all functions needed to be individually implemented and adapted to the Vulkan API. For all requested SYCL objects we create equivalent Vulkan objects, as summarized in table 2 . We currently target the Vulkan API Version 1.1 and require extensions VK_KHR_timeline_semaphore as well as VK_KHR_shader_float16_int8.

VK_KHR_timeline_semaphore enables timeline semaphores, the only synchronization option that offers unified support for host-todevice, device-to-host and device-to-device synchronization. To use 8-bit integer values in a kernel, which could happen rather easily by using variables of character type in $\mathrm{C}++_{+}$, we also include VK_KHR_shader_float16_int8 by default. Since both extensions are promoted in Vulkan 1.2 neither represents a significant limitation.

Without describing the entire implementation in detail, we want to highlight some of the more challenging aspects. One such challenge was the correct handling of buffers, while fulfilling the low latency and high bandwidth needs of highly parallel programs. This generally requires allocating buffer memory on the device, which is then often not directly accessible by host code. A common portable approach is to use a staging buffer on the host side, where memory can be updated and afterwards copied efficiently onto the related device buffer, or vice versa for reading. As the SYCL runtime only maintains a single buffer object, without knowledge about where data is actually stored, we encapsulate the staging process within our plugin.

A common case is that the SYCL runtime already allocated host memory for a buffer object, and requests that the plugin reuses that memory for the API. For our prototype implementation we 


\begin{tabular}{|c|c|c|}
\hline Benchmark & Nvidia GTX 1070 & Intel HD Graphics 530 \\
\hline Stencil & $\begin{array}{l}5000 \text { timesteps, } 8192 * 8192 \text { floating point elements } \\
\text { on workgroups of size } 32 * 32\end{array}$ & $\begin{array}{l}5000 \text { timesteps, } 4096 * 4096 \text { floating point elements } \\
\text { on workgroups of size } 16 * 16\end{array}$ \\
\hline MatMul & $\begin{array}{l}8192 * 8192 \text { floating point elements on workgroups } \\
\text { of size } 32 * 32\end{array}$ & $\begin{array}{l}4096 * 4096 \text { floating point elements on workgroups } \\
\text { of size } 16 * 16\end{array}$ \\
\hline PrefixSum & 4194304 elements & 262144 elements \\
\hline
\end{tabular}

Table 3: Individual test configurations for each benchmark and device combination.

encountered some issues with importing memory to Vulkan. First, importing requires the VK_EXT_external_memory_host extension, which may not be available, and secondly it has certain alignment requirements. On all our tested hardware Vulkan needs a $4 \mathrm{kB}$ alignment and we therefore changed the default alignment for buffers to accommodate this requirement. However, in SYCL, it is possible for the programmer to allocate host memory arbitrarily and lend ownership to the runtime when creating a buffer. In case the aforementioned extension is not available or insufficiently aligned memory is used, Sylkan creates a dedicated staging buffer. This has the drawback that the data is replicated within the SYCL runtime, the staging buffer and in device memory, but it ensures broader hardware and software support.

For larger programs, the DPC ++ compiler allows each kernel to generate its own SPIR-V binary and finally bundles them into a single fat binary. However, the SYCL runtime has no further metainformation about these binaries and it cannot discriminate which kernel was bundled into which binary. It solves this dilemma by delegating it and passing all binaries to all available plugins. Each plugin must then be able to identify compatible binaries by some bundle information or by attempting to parse the binary itself. To support this use case, the Sylkan plugin includes the LLVMSPIRV library for parsing SPIR-V binaries.

\section{EVALUATION}

\subsection{Testing Setup}

To evaluate the performance of our Sylkan prototype we chose three simple benchmarks covering various aspects of kernel and runtime system performance, and created a SYCL and native Vulkan version of each of them for comparison purposes ${ }^{6}$.

Stencil implements an iterative 2D heat stencil computation to explore runtime system performance with many successive kernel calls.

MatMul is a basic dense matrix-matrix multiplication with a single kernel call designed to determine whether we suffer from any fundamental code generation inefficiencies.

PrefixSum is our final benchmark and shows the behaviour of two distinct interacting kernels.

Two GPUs were benchmarked, a dedicated Nvidia GTX 1070 (Driver Version 461.09) and an integrated Intel HD Graphics 530 (Driver Version 27.20.100.9126). Both are installed in the same desktop PC running Windows 10 . We compare three implementations, namely

\footnotetext{
${ }^{6}$ https://github.com/tadeaustria/SYCLBenchmarks/tree/syclcon2021
}

Sylkan, DPC++ based on OpenCL 3.0, and Vulkan native. Since Nvidia does not support OpenCL beyond 1.x, we were only able to measure Vulkan native and Sylkan results on this platform. For each combination we tracked both the pure kernel time as well as the complete host time from initialization to the availability of the result in host memory. We performed five measurements each and report the mean.

Table 3 lists the specific test configuration for each benchmark and hardware platform.

\subsection{Performance}

Figure 5 and fig. 7 show the kernel and host results on the Nvidia platform, and fig. 6 and fig. 8 do the same on the Intel integrated GPU. To enable effective performance comparisons across different benchmarks with distinct absolute execution time, we display relative time compared to the native Vulkan baseline implementation in our charts. A value lower/higher than 1 means faster/slower than the native Vulkan implementation. We have also included error bars based on standard deviation in our charts for completeness, however they are barely visible as we encountered only very minor variance across benchmark runs.

Figure 5 and fig. 6 show that the kernel time required by Sylkan is generally comparable to, but somewhat slower than the native Vulkan implementation as well as the OpenCL-based SYCL implementation. Larger performance gaps, such as in the matrix multiplication example on Nvidia, relate to how additional variables required for index calculations are passed and accessed as buffers in our current implementation, which appears to reduce the toolchain's ability to optimize these operations.

Of particular note is the PrefixSum result on our Nvidia test platform. Here, Sylkan shows faster kernel execution even though the overall host time is slower. We investigated the SPIR-V generated in each case and noticed that for this particular benchmark, the code generated by Sylkan is actually slightly more concise than the native Vulkan equivalent, saving two integer instructions and a memory operation. One reason for that could be that the Clang and LLVM toolchain of the Sylkan implementation performs some optimizations on LLVM IR level that the GLSL toolchain omits. Nevertheless, the performance impact of this code difference appears to be negligible on the Intel platform.

Figure 7 and fig. 8 illustrate that the differences in host performance largely correlate with the kernel times, especially for matrix multiplication. The most significant divergence from the results of the kernel-only benchmarks can be observed for PrefixSum. This 


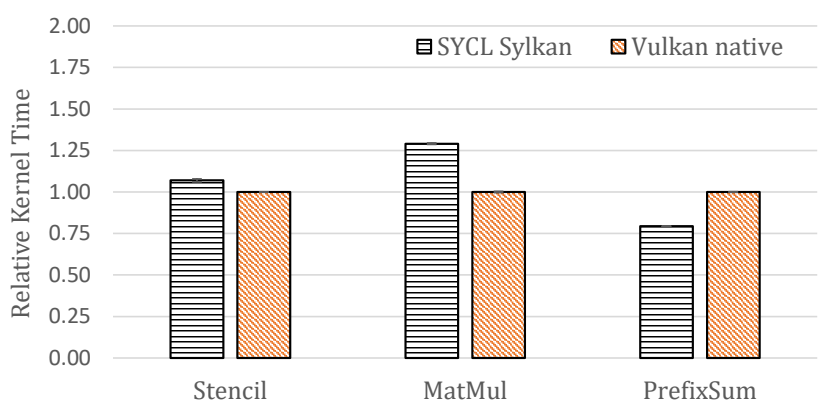

Figure 5: Relative Kernel Time on Nvidia GeForce GTX 1070

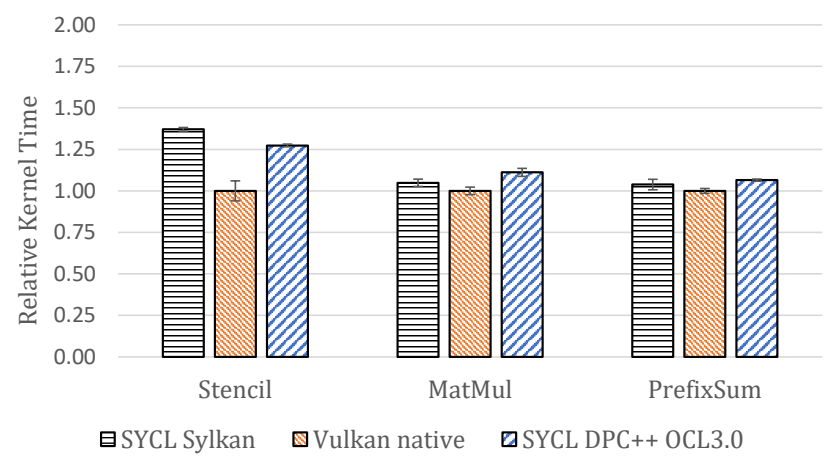

Figure 6: Relative Kernel Time on Intel(R) HD Graphics 530

benchmark is by far the shortest in terms of absolute time - below one millisecond - which means that even minor startup or allocation overhead in the runtime is amplified significantly. This affected Sylkan on both the Nvidia and Intel platforms relative to native Vulkan, but is particularly noticeable with the OpenCL-based implementation, which appears to incur a much larger initialization overhead. However, the practical relevance of this issue to any real-world applications should be far less than what is indicated by this comparison, as the additional time in the millisecond range is mitigated by a larger overall workload. Still, it is noteworthy that Sylkan benefits from the relatively low Vulkan overhead in this particular test.

Relative host times for the Stencil benchmark also increase for Sylkan, especially on the Intel platform. This makes sense as the large number of iterations results in far more host-device interactions compared to the other benchmarks. This points to some expected potential for optimizing the parameter passing scheme and synchronization in the current Sylkan runtime plugin.

Overall, although the current Sylkan prototype implementation has some performance drawbacks, we believe that our results indicate that this approach is viable and can achieve performance

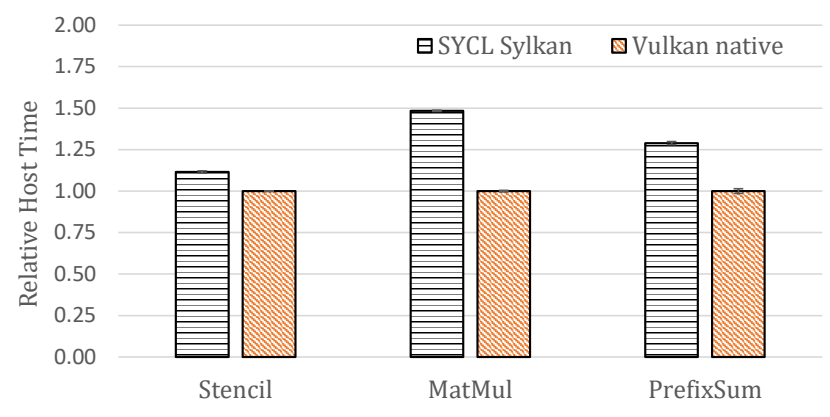

Figure 7: Relative Host Time on Nvidia GeForce GTX 1070

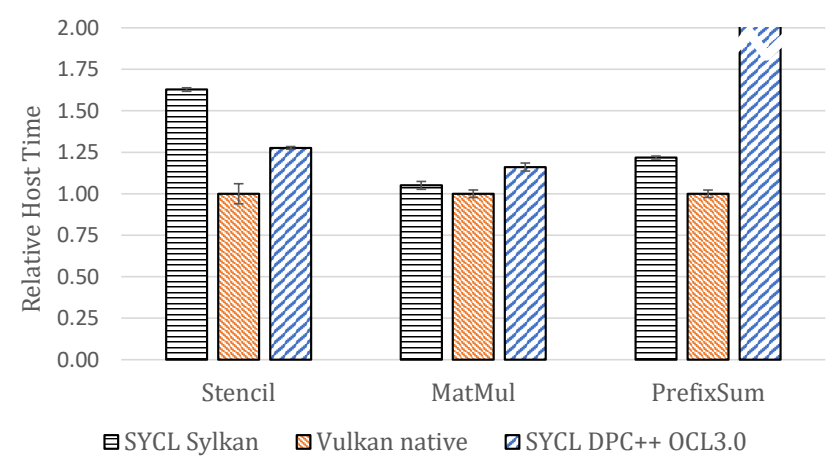

Figure 8: Relative Host Time on Intel(R) HD Graphics 530

comparable to other backends with a more mature implementation. This in turn also means that it is possible to maintain the programmability advantages of SYCL while targeting Vulkan devices, without any code-level changes.

\subsection{Feature Coverage}

As the basis of our feature evaluation we rely on the Intel LLVM test suite $^{7}$, which extends the LLVM test suite by SYCL tests. Running this test suite on our testing platform results in a success rate of $48.6 \%$, with 123 out of 253 tests passed and an additional 147 unsupported. Some of the failed tests target optional or new SYCL 2020 features such as unified shared memory (USM), while others relate to more basic features not yet implemented in Sylkan. In this section we will summarize the most significant feature gaps of the prototype and describe possible issues and solutions.

The first category of failures relates to image and sampler support. We skipped this entire category of the API purely due to time and manpower constraints, since we believe that there are no significant research or compilation challenges to solve in order to enable

\footnotetext{
${ }^{7}$ https://github.com/intel/llvm-test-suite
} 
these features. If such support is required, the related code generation in the frontend compiler must be checked and potentially extended by some straightforward mappings, and all related API calls in the plugin need to be implemented.

Far more importantly, the basic feature of local memory support is also currently missing from our prototype. The difficulties here relate to the implementation of argument passing, detailed in section 3.1.2. As opposed to OpenCL, where local memory only requires a size as an argument and can be used as a pointer within the kernel, the Vulkan API requires generating an array in local memory in the SPIR-V code. Furthermore, the size of this array must be known at compile time. A possible implementation path would be using specialization constants within Vulkan, however, this necessitates a significant redesign of the general argument passing implementation in the current toolchain. As this missing feature significantly limits the applicability of the current prototype, as well as its ability to leverage the full performance potential of many accelerator devices, it is a high-priority target for future work.

Another missing feature category are SYCL stream objects for printing information. This feature requires the ability to partition buffers into sub-buffers, and no trivial mapping to Vulkan is possible. As SYCL streams appear to be more commonly used for debugging purposes in practice, we have not prioritized their implementation for now. Debugging the kernel on host devices or using alternative backends is a possible workaround at this point in time.

A more minor restriction is that offsets in the Vulkan API, when launching compute shaders, work only in multiples of the work group size. If more flexible offset functionality is required, it could be implemented as an extra mapping layer in the kernel code. Another related difference compared to OpenCL is that Vulkan only supports 4-byte integers as invocation indices. Especially for 1-D shader executions this limits the computation to 4,294,967,295 work items and is therefore more restrictive. SYCL specifies the type of indices as size_t and on 64-bit systems the type would support larger indices than supported by our current prototype. Again, this limitation could be circumvented in the future - at least for 1D Kernels where it is most likely to occur in practice - by distributing the invocation on 2 dimensions instead and reconstructing a single 64 bit index in the kernel.

A final quality-of-implementation issue of the current prototype is that some required changes of SYCL headers prevent the original feature of compiling one program for multiple devices. Even though device code compilation is split for different targets, there is only one integration header generation routine. Since we require additional information in these headers (see section 3.1.2), they are mutually incompatible. It is still possible to generate separate executables for each target, but not a single binary targeting both Vulkan and other backends. This is another instance where the source of the issue is not a fundamental semantic mismatch, and which could be resolved by investing more engineering effort into the implementation.

\section{RELATED WORK}

Some of the most directly related work to Sylkan are other SYCL implementations, however those were already covered in detail in section 2.3. Each solves similar problems, and we have reused a significant amount of work from both the LLVM [12] framework in general and Intel's DPC++ implementation [3] in particular. However, targeting Vulkan compute as a backend introduces several unique challenges that are the main focus of this paper.

In a similar vein, there was significant prior interest in tools that simplify or fully automate the porting of existing programs written using vendor-specific APIs - primarily CUDA, due to its widespread popularity - to different architectures and interfaces. An early support tooling approach was presented by Harvey and De Fabritiis [9], and Perkins went a step further in automation by introducing CUDA-on-CL [17]. These tools are conceptually similar to Sylkan in their goal, but as both the theoretical semantics of the languages being mapped as well as their respective tool ecosystems differ significantly, there is relatively little overlap in practice.

A slightly different approach, targeting the same overarching goal of increasing the portability of CUDA applications, is followed by AMD's Heterogeneous-Computing Interface for Portability (HIP) [2]. It offers a C++ language dialect which abstracts over both Nvidia and AMD backends, and includes tooling for assisted conversion of CUDA applications to this language. Kuznetsov et al. provide an experience and performance report [11] on applying this technology to molecular dynamics code. With HIPCL [4], Babej et al. close the circle by presenting an implementation of HIP which targets OpenCL devices supporting SPIR-V.

It is clear from all of these efforts over the years that better support for high-level cross-vendor compute platforms is very desirable. SYCL is, to some extent, a direct response to this demand, and our goal with Sylkan is to show that its applicability can be further extended to an additional category of devices by targeting Vulkan compute as its backend.

A different category of related work involves the use of the Vulkan API's compute capabilities for non-graphical tasks. Gunadi et al. implemented real-time SPH fluid simulation on GPUs with Vulkan compute shaders [8], while Gkeka et al. performed a broader evaluation of the comparative performance of compute algorithms implemented in Vulkan [7]. With Vcomputebench [13], Mammeri et al. introduced a benchmark suite specifically targeted at characterizing Vulkan compute performance on embedded devices. These works illustrate that Vulkan is a viable target even for pure compute applications.

Thoman et al. recently leveraged Vulkan for room response simulation [25]. In this case it is particularly noteworthy from a Sylkan perspective that the algorithm benefits from hardware raytracing acceleration, which is not currently natively available in any of the more compute-focused APIs. Making it easier to access or interoperate with more niche features such as this is another potential advantage of a SYCL implementation based on Vulkan.

\section{CONCLUSION AND FUTURE WORK}

In this paper, we have investigated the high-level semantic differences between SYCL and Vulkan compute, the engineering gap between their respective tool ecosystems, and presented a compiler and runtime approach for bridging this gap. We demonstrated and validated our approach by implementing Sylkan, a novel SYCL platform targeting Vulkan compute devices. An initial performance 
evaluation with three benchmarks, each implemented both as SYCL and as native Vulkan applications, was performed on two hardware architectures. While some performance penalty exists - as is to be expected from a scientific prototype - the overall results prove the viability of our approach for achieving performance comparable to native Vulkan and OpenCL-based SYCL implementations. We also evaluated the quality of our implementation, categorized its current feature gaps, and discussed how each of them could be resolved in the future.

While we present a functional implementation covering the full toolchain, including compilation and a runtime system, a large amount of future work remains in order to fully realize and demonstrate the potential of Vulkan-backed SYCL implementations. There are three major categories of such work: optimizing the compiler toolchain and runtime system in order to improve performance, extending the SYCL feature coverage offered by Sylkan, and evaluating its performance on additional hardware devices and with more large-scale benchmarks.

\section{ACKNOWLEDGMENTS}

This research was partially funded by the FFG (Austrian Research Promotion Agency) project INPACT (project number 868018), as well as the FWF as part of the CELERITY project (I 3388).

\section{REFERENCES}

[1] Aksel Alpay and Vincent Heuveline. 2020. SYCL beyond OpenCL: The architecture, current state and future direction of hipSYCL. In Proceedings of the International Workshop on OpenCL. 1-1.

[2] AMD. 2020. HIP Programming Guide v4.0 - ROCm Documentation. https: $/ /$ rocmdocs.amd.com/en/latest

[3] Ben Ashbaugh, Alexey Bader, James Brodman, Jeff Hammond, Michael Kinsner, John Pennycook, Roland Schulz, and Jason Sewall. 2020. Data Parallel C++ Enhancing SYCL Through Extensions for Productivity and Performance. In Proceedings of the International Workshop on OpenCL. 1-2.

[4] Michal Babej and Pekka Jääskeläinen. 2020. HIPCL: Tool for Porting CUDA Applications to Advanced OpenCL Platforms Through HIP. In Proceedings of the International Workshop on OpenCL. 1-3.

[5] Rachel Courtland. 2015. Gordon Moore: The Man Whose Name Means Progress. https://spectrum.iee.org/computing/hardware/gordon-moore-theman-whose-name-means-progress

[6] Jianbin Fang, Chun Huang, Tao Tang, and Zheng Wang. 2020. Parallel programming models for heterogeneous many-cores: a comprehensive survey. CCF Transactions on High Performance Computing 2, 4 (2020), 382-400.

[7] Maria Rafaela Gkeka, Nikolaos Bellas, and Christos D Antonopoulos. 2019. Comparative Performance Analysis of Vulkan Implementations of Computational Applications. In Proceedings of the International Workshop on OpenCL. 1-1.

[8] Samuel I Gunadi and Pujianto Yugopuspito. 2018. Real-Time GPU-based SPH Fluid Simulation Using Vulkan and OpenGL Compute Shaders. In 2018 4th International Conference on Science and Technology (ICST). IEEE, 1-6.

[9] Matt J Harvey and Gianni De Fabritiis. 2011. Swan: A tool for porting CUDA programs to OpenCL. Computer Physics Communications 182, 4 (2011), 1093 1099.

[10] Ronan Keryell, Ruyman Reyes, and Lee Howes. 2015. Khronos SYCL for OpenCL: a tutorial. In Proceedings of the 3rd International Workshop on OpenCL. 1-1.

[11] Evgeny Kuznetsov and Vladimir Stegailov. 2019. Porting CUDA-Based Molecular Dynamics Algorithms to AMD ROCm Platform Using HIP Framework: Performance Analysis. 121-130. https://doi.org/10.1007/978-3-030-36592-9_11

[12] Chris Lattner and Vikram Adve. 2004. LLVM: A compilation framework for lifelong program analysis \& transformation. In International Symposium on Code Generation and Optimization, 2004. CGO 2004. IEEE, 75-86.

[13] Nadjib Mammeri and Ben Juurlink. 2018. Vcomputebench: A vulkan benchmark suite for gpgpu on mobile and embedded gpus. In 2018 IEEE International Symposium on Workload Characterization (IISWC). IEEE, 25-35.

[14] Anne Meade, Deva Kumar Deeptimahanti, Jim Buckley, and J. J. Collins. 2017. An empirical study of data decomposition for software parallelization. Fournal of Systems and Software 125 (2017), 401-416.

[15] Tianyun Ni. 2009. Direct Compute: Bring GPU computing to the mainstream. In GPU technology conference. 23.
[16] NVIDIA, Péter Vingelmann, and Frank H.P. Fitzek. 2020. CUDA, release: 10.2.89. https://developer.nvidia.com/cuda-toolkit

[17] Hugh Perkins. 2017. CUDA-on-CL: a compiler and runtime for running NVIDIA ${ }^{\circledR}$ CUDA $^{\mathrm{TM}} \mathrm{C}++11$ applications on OpenCL ${ }^{\mathrm{TM}} 1.2$ Devices. In Proceedings of the 5th International Workshop on OpenCL. 1-4.

[18] Ruyman Reyes, Gordon Brown, Rod Burns, and Michael Wong. 2020. SYCL 2020: More than meets the eye. In Proceedings of the International Workshop on OpenCL. Association for Computing Machinery, New York, NY, USA, 1-1.

[19] Graham Sellers and John Kessenich. 2016. Vulkan programming guide: The official guide to learning vulkan. Addison-Wesley Professional.

[20] Dave Shreiner, Graham Sellers, John Kessenich, and Bill Licea-Kane. 2013. OpenGL programming guide: The Official guide to learning OpenGL, version 4.3. AddisonWesley.

[21] The Khronos Group. 2012. The OpenCL Specification, Version 1.2 Revision 19. https://www.khronos.org/registry/OpenCL/specs/opencl-1.2.pdf

[22] The Khronos Group. 2014. The SPIR Specification, Version 1.2. https://www. khronos.org/registry/SPIR/specs/spir_spec-1.2.pdf

[23] The Khronos Group. 2018. SYCL Specification, Version 1.2.1 Revision 3. https: //www.khronos.org/registry/SYCL/specs/sycl-1.2.1.pdf

[24] The Khronos Group. 2020. Vulkan 1.2.166 Specification. https://www.khronos. org/registry/vulkan/specs/1.2/pdf/vkspec.pdf

[25] Peter Thoman, Markus Wippler, Robert Hranitzky, and Thomas Fahringer. 2020. RTX-RSim: Accelerated Vulkan Room Response Simulation for Time-of-Flight Imaging. In Proceedings of the International Workshop on OpenCL. Association for Computing Machinery, New York, NY, USA. 